\title{
Subcritical polarisations of symplectic manifolds have degree one
}

\author{
Hansjörg Geiges(D, Kevin Sporbeck, And Kai Zehmischid
}

\begin{abstract}
We show that if the complement of a Donaldson hypersurface in a closed, integral symplectic manifold has the homology of a subcritical Stein manifold, then the hypersurface is of degree one. In particular, this demonstrates a conjecture by Biran and Cieliebak on subcritical polarisations of symplectic manifolds. Our proof is based on a simple homological argument using ideas of Kulkarni-Wood.
\end{abstract}

Mathematics Subject Classification. 53D35, 57R17, 57R19, 57R95.

Keywords. Donaldson hypersurface, Subcritical polarisation, KulkarniWood homomorphism.

1. Donaldson hypersurfaces and symplectic polarisations. Let $(M, \omega)$ be a closed, connected, integral symplectic manifold, that is, the de Rham cohomology class $[\omega]_{\mathrm{dR}}$ lies in the image of the homomorphism $H^{2}(M) \rightarrow H_{\mathrm{dR}}^{2}(M)=$ $H^{2}(M ; \mathbb{R})$ induced by the inclusion $\mathbb{Z} \rightarrow \mathbb{R}$. The cohomology classes in $H^{2}(M)$ mapping to $[\omega]_{\mathrm{dR}}$ are called integral lifts, and by abuse of notation, we shall write $[\omega]$ for any such lift. Following McDuff and Salamon [11, Section 14.5], we call a codimension 2 symplectic submanifold $\Sigma \subset M$ a Donaldson hypersurface if it is Poincaré dual to $d[\omega] \in H^{2}(M)$ for some integral lift $[\omega]$ and some (necessarily positive) integer $d$. Donaldson [5] has established the existence of such hypersurfaces for any sufficiently large $d$.

The pair $(M, \Sigma)$ is called a polarisation of $(M, \omega)$, and the number $d \in \mathbb{N}$, the degree of the polarisation. Biran and Cieliebak [2] studied these polarisations in the Kähler case, where $\omega$ admits a compatible integrable almost complex structure $J$. In that setting, the complement $(M \backslash \Sigma, J)$ admits in a natural way the structure of a Stein manifold.

This research is part of a project in the SFB/TRR 191 Symplectic Structures in Geometry, Algebra and Dynamics, funded by the DFG (Project-ID 281071066-TRR 191). 
As shown recently by Giroux [8], building on work of Cieliebak-Eliashberg, even in the non-Kähler case the complement of a symplectic hypersurface $\Sigma \subset M$ found by Donaldson's construction admits the structure of a Stein manifold. Here, of course, the complex structure on $M \backslash \Sigma$ does not, in general, extend over $\Sigma$. Complements of Donaldson hypersurfaces are also studied in $[4]$.

2. Subcritical polarisations. The focus of Biran and Cieliebak [2] lay on $s u b$ critical polarisations of Kähler manifolds, which means that $(M \backslash \Sigma, J)$ admits a plurisubharmonic Morse function $\varphi$ all of whose critical points have, for $\operatorname{dim} M=2 n$, index less than $n$. (They also assumed that $\varphi$ coincides with the plurisubharmonic function defining the natural Stein structure outside a compact set containing all critical points of $\varphi$.)

More generally, McDuff and Salamon [11, p. 504] propose the study of polarisations $(M, \Sigma)$ where the complement $M \backslash \Sigma$ is homotopy equivalent to a subcritical Stein manifold (of finite type). We relax this condition a little further and call $(M, \Sigma)$ homologically subcritical if $M \backslash \Sigma$ has the homology of a subcritical Stein manifold, that is, of a CW-complex containing finitely many cells up to dimension at most $n-1$. This means that there is some $\ell \leq n-1$ such that $H_{k}(M \backslash \Sigma)$ vanishes for $k \geq \ell+1$ and $H_{\ell}(M \backslash \Sigma)$ is torsion-free.

Motivated by the many examples they could construct, Biran and Cieliebak [2, p. 751] conjectured that subcritical polarisations necessarily have degree 1. They suggested an approach to this conjecture using either symplectic or contact homology. A rough sketch of a proof along these lines, in the language of symplectic field theory, was given by Eliashberg-Givental-Hofer [6, p. 661]. A missing assumption $c_{1}(M \backslash \Sigma)=0$ of that argument and a few more detailsstill short of a complete proof-were added by J. He [9, Proposition 4.2], who appeals to Gromov-Witten theory and polyfolds.

Here is our main result, which entails the conjecture of Biran-Cieliebak.

Theorem 1. Let $(M, \omega)$ be a closed, integral symplectic manifold, and $\Sigma \subset M a$ compact symplectic submanifold of codimension 2, Poincaré dual to the integral cohomology class $d[\omega]$ for some (positive) integer $d$. If $(M, \Sigma)$ is homologically subcritical, then $d[\omega] /$ torsion is indivisible in $H^{2}(M) /$ torsion. In particular, $d=1$.

Notation. The free part of $H^{2}(M)$ is not a well-defined subgroup of $H^{2}(M)$, but the torsion part is, and so is the quotient group $H^{2}(M) /$ torsion, which is free abelian. We write $d[\omega] /$ torsion for the class represented by $d[\omega]$ in this quotient. This class is determined by $[\omega]_{\mathrm{dR}}$ and does not depend on the choice of integral lift $[\omega]$.

Our proof is devoid of any sophisticated machinery. The assumption on $(M, \Sigma)$ to be homologically subcritical guarantees the surjectivity of a certain homomorphism in homology described by Kulkarni and Wood [10]; this implies the claimed indivisibility.

3. The Kulkarni-Wood homomorphism. We consider a pair $(M, \Sigma)$ consisting of a closed, connected, oriented manifold $M$ of dimension $2 n$, and a compact, 
oriented hypersurface $\Sigma \subset M$ of codimension 2. No symplectic assumptions are required in this section.

Write $i: \Sigma \rightarrow M$ for the inclusion map. The Poincaré duality isomorphisms on $M$ and $\Sigma$ from cohomology to homology, given by capping with the fundamental class, are denoted by $\mathrm{PD}_{M}$ and $\mathrm{PD}_{\Sigma}$, respectively.

In their study of the topology of complex hypersurfaces, Kulkarni and Wood [10] used the following composition, which we call the Kulkarni-Wood homomorphism:

$$
\begin{gathered}
\Phi_{\mathrm{KW}}: H^{k}(M) \stackrel{i^{*}}{\longrightarrow} H^{k}(\Sigma) \stackrel{\mathrm{PD}_{\Sigma}}{\longrightarrow} H_{2 n-2-k}(\Sigma) \stackrel{i_{*}}{\longrightarrow} \\
\stackrel{i_{*}}{\longrightarrow} H_{2 n-2-k}(M) \stackrel{\mathrm{PD}_{M}^{-1}}{\longrightarrow} H^{k+2}(M) .
\end{gathered}
$$

Lemma 2. The Kulkarni-Wood homomorphism equals the cup product with the cohomology class $\sigma:=\operatorname{PD}_{M}^{-1}\left(i_{*}[\Sigma]\right) \in H^{2}(M)$.

Proof. For $\alpha \in H^{k}(M)$, we compute

$$
\begin{aligned}
\Phi_{\mathrm{KW}}(\alpha) & =\mathrm{PD}_{M}^{-1} i_{*} \mathrm{PD}_{\Sigma} i^{*} \alpha=\mathrm{PD}_{M}^{-1} i_{*}\left(i^{*} \alpha \cap[\Sigma]\right) \\
& =\mathrm{PD}_{M}^{-1}\left(\alpha \cap i_{*}[\Sigma]\right)=\mathrm{PD}_{M}^{-1}\left(\alpha \cap \mathrm{PD}_{M}(\sigma)\right) \\
& =\mathrm{PD}_{M}^{-1}(\alpha \cap(\sigma \cap[M]))=\mathrm{PD}_{M}^{-1}((\alpha \cup \sigma) \cap[M]) \\
& =\alpha \cup \sigma ;
\end{aligned}
$$

all the formulae for cup and cap products used in this chain of identities can be found in [3, Theorem VI.5.2].

Lemma 3. If the complement $M \backslash \Sigma$ has the homology type of a $C W$-complex of dimension $\ell$ for some $\ell \leq n-1$, then $\Phi_{\mathrm{KW}}: H^{k}(M) \rightarrow H^{k+2}(M)$ is surjective in the range $\ell-1 \leq k \leq 2 n-\ell-2$.

Proof. Write $\nu \Sigma$ for an open tubular neighbourhood of $\Sigma$ in $M$. By homotopy, excision, duality, and the universal coefficient theorem, we have

$$
\begin{aligned}
H_{k}(M, \Sigma) & \cong H_{k}(M, \nu \Sigma) \cong H_{k}(M \backslash \nu \Sigma, \partial(\nu \Sigma)) \\
& \cong H^{2 n-k}(M \backslash \nu \Sigma) \\
& \cong F H_{2 n-k}(M \backslash \Sigma) \oplus T H_{2 n-k-1}(M \backslash \Sigma),
\end{aligned}
$$

where $F$ and $T$ denote the free and the torsion part, respectively. This vanishes for $2 n-k-1 \geq \ell$, that is, for $k \leq 2 n-\ell-1$. It follows that the homomorphism $i_{*}: H_{2 n-2-k}(\Sigma) \rightarrow H_{2 n-2-k}(M)$ is surjective for $2 n-2-k \leq 2 n-\ell-1$, or $k \geq \ell-1$.

Similarly (or directly by the Poincaré-Lefschetz duality), we have

$$
H^{k}(M, \Sigma) \cong H_{2 n-k}(M \backslash \Sigma),
$$

which vanishes for $2 n-k \geq \ell+1$, that is, for $k \leq 2 n-\ell-1$. Hence, the homomorphism $i^{*}: H^{k}(M) \rightarrow H^{k}(\Sigma)$ is surjective for $k+1 \leq 2 n-\ell-1$, that is, for $k \leq 2 n-\ell-2$. 
4. Proof of Theorem 1. Under the assumptions of Theorem 1, the homomorphism $\Phi_{\mathrm{KW}}: H^{k}(M) \rightarrow H^{k+2}(M)$ is surjective at least in the range $n-2 \leq k \leq n-1$; simply set $\ell=n-1$ in Lemma 3 . Thus, we can pick an even number $k=2 m$ in this range. The free part of $H^{2 m+2}(M)$ is nontrivial since this cohomology group contains the element $[\omega]^{m+1}$ of infinite order.

On the other hand, $\Phi_{\mathrm{KW}}$ is given by the cup product with $d[\omega]$, as shown in Lemma 2, so we have the commutative diagram

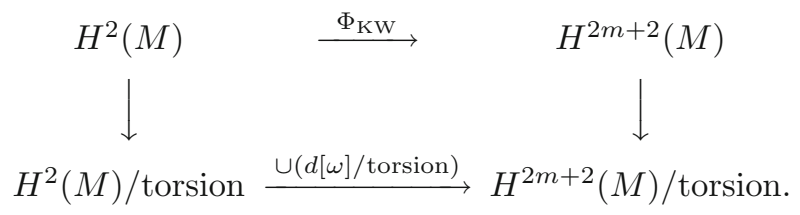

If $d[\omega] /$ torsion were divisible, the map on free abelian groups at the bottom of this diagram would not be surjective, and neither would be $\Phi_{\mathrm{KW}}$.

Remark 4. The real Euler class of the circle bundle $\partial(\nu \Sigma)$ equals $d[\omega]_{\mathrm{dR}}$, and the natural Boothby-Wang contact structure on this bundle has an exact convex filling by the complement $M \backslash \nu \Sigma$, see [7, Lemma 3], [8, Proposition 5], and [4, Lemma 2.2]. With [1, Theorem 1.2], the condition 'homologically subcritical' of Theorem 1 may be replaced by assuming the existence of some subcritical Stein filling of this Boothby-Wang contact structure.

Funding Open Access funding enabled and organized by Projekt DEAL.

Open Access. This article is licensed under a Creative Commons Attribution 4.0 International License, which permits use, sharing, adaptation, distribution and reproduction in any medium or format, as long as you give appropriate credit to the original author(s) and the source, provide a link to the Creative Commons licence, and indicate if changes were made. The images or other third party material in this article are included in the article's Creative Commons licence, unless indicated otherwise in a credit line to the material. If material is not included in the article's Creative Commons licence and your intended use is not permitted by statutory regulation or exceeds the permitted use, you will need to obtain permission directly from the copyright holder. To view a copy of this licence, visit http://creativecommons.org/ licenses/by/4.0/.

Publisher's Note Springer Nature remains neutral with regard to jurisdictional claims in published maps and institutional affiliations.

\section{References}

[1] Barth, K., Geiges, H., Zehmisch, K.: The diffeomorphism type of symplectic fillings. J. Symplect. Geom. 17, 929-971 (2019)

[2] Biran, P., Cieliebak, K.: Symplectic topology on subcritical manifolds. Comment. Math. Helv. 76, 712-753 (2001)

[3] Bredon, G.E.: Topology and Geometry. Graduate Texts in Mathematics, vol. 139. Springer, Berlin (1993) 
[4] Diogo, L., Lisi, S.T.: Symplectic homology of complements of smooth divisors. J. Topol. 12, 967-1030 (2019)

[5] Donaldson, S.K.: Symplectic submanifolds and almost-complex geometry. J. Differential Geom. 44, 666-705 (1996)

[6] Eliashberg, Y., Givental, A., Hofer, H.: Introduction to symplectic field theory. Geom. Funct. Anal. 2000 (Special Volume, Part II), 560-673 (2000)

[7] Geiges, H., Stipsicz, A.I.: Contact structures on product five-manifolds and fibre sums along circles. Math. Ann. 348, 195-210 (2010)

[8] Giroux, E.: Remarks on Donaldson's symplectic submanifolds. Pure Appl. Math. Q. 13, 369-388 (2017)

[9] He, J.: Correlators and descendants of subcritical Stein manifolds. Internat. J. Math. 24, 1350004 (2013)

[10] Kulkarni, R.S., Wood, J.W.: Topology of nonsingular complex hypersurfaces. Adv. Math. 35, 239-263 (1980)

[11] McDuff, D., Salamon, D.: Introduction to Symplectic Topology. Oxford Graduate Texts in Mathematics, vol. 27, 3rd edn. Oxford University Press, Oxford (2017)

HANsJörg GEIGES

Mathematisches Institut

Universität zu Köln

Weyertal 86-90

50931 Köln

Germany

e-mail: geiges@math.uni-koeln.de

Kevin Sporbeck and Kai Zehmisch

Fakultät für Mathematik

Ruhr-Universität Bochum

Universitätsstraße 150

44780 Bochum

Germany

e-mail: kevin.sporbeck@rub.de

e-mail: kai.zehmisch@rub.de

Received: 4 February 2021

Revised: 23 February 2021

Accepted: 15 March 2021. 